\title{
Purificación del Biogás para la producción de Biometano, a través de sistemas de filtración de $\mathrm{CO} 2$ y vapor de agua.
}

Biogas purification for the production of Biomethane, through $\mathrm{CO} 2$ and water vapor filtration systems.

\author{
Godofredo Peña Dávila ${ }^{\mathrm{a}}$ y Gonzalo Dávila-del-Carpio ${ }^{\mathrm{b}}$ \\ ${ }^{a}$ Instituto de Investigación y Desarrollo para el Sur (IIDS). Arequipa Perú. \\ bUniversidad Católica de Santa María. Arequipa Perú.
}

\section{INFORMACIÓN}

\section{Historia del Artículo}

Recepción: 10/06/2019

Revisión: 05/05/2020

Aceptación: 20/05/2020

\section{Palabras Clave}

Biogás, biometano, biomasa, energías renovables, Scrubber, PSA, filtración, dióxido de carbono, vapor de agua

\section{Key Words}

Biogás, biomethane, biomass, renewable energy, Scrubber, PSA, filtration, dioxide carbon, water steam

\section{DOI}

https://doi.org/10.35286/veritas. v21i 2.277

\begin{abstract}
RESUMEN
El biogás es un biocombustible producto de la digestión anaeróbica de productos orgánicos. Su poder calorífico es bajo (18 MJ/Nm3) y mejora cuando se convierte en biometano por eliminación de $\mathrm{CO} 2$, vapor de agua y $\mathrm{H} 2 \mathrm{~S}$. Nos propusimos desarrollar tecnología para purificar el biogás obtenido en la planta HPTC del Fundo La Católica de El Pedregal-Majes, para lo cual se diseñó, construyó y evaluó un sistema de filtrado por depuración con agua presurizada (Scrubber), para reducir el contenido de $\mathrm{CO} 2$ a valores menores a $5.0 \% \mathrm{~V} / \mathrm{V}$; para ello trabajamos a 03 presiones de operación $(6,9$ y $10 \mathrm{bar})$ combinadas con 03 temperaturas $\left(10,15\right.$ y $\left.20^{\circ} \mathrm{C}\right)$, obteniéndose el mejor resultado de purificación, $4,3 \% \mathrm{~V} / \mathrm{V}$ de $\mathrm{CO} 2$ a $10^{\circ} \mathrm{C}$ y $10 \mathrm{bar}$ de presión. El sistema toma el biogás, lo presuriza e introduce por un tubo de acero de $0.05 \mathrm{~m}$ de diámetro y $3,0 \mathrm{~m}$ de altura ubicado en la parte inferior, en cuyo interior se encuentran 04 tubos de PVC de diámetros decrecientes, con perforaciones y separadores que aumentan la superficie de contacto y por ende la absorción del CO2. Igualmente, se desarrolló un sistema de filtrado PSA para el vapor de agua; se presuriza el biogás e introduce en la parte inferior por un tubo de acero de $0,2 \mathrm{~m}$ de diámetro y $0,5 \mathrm{~m}$ de altura, en cuyo interior se encuentra la zeolita como adsorbente. El flujo de biogás es controlado a través de válvulas y el filtrado se retira por la parte superior, logrando reducir el contenido de vapor de agua a $2.2 \% \mathrm{~V} / \mathrm{V}$ a 8 bar de presión y temperatura ambiente.
\end{abstract}

\begin{abstract}
Biogas is a biofuel, product of anaerobic digestion of organic products. Its calorific power is low (18 MJ/Nm3) and improves when it is converted to biomethane by elimination of $\mathrm{CO} 2$, water steam and $\mathrm{H} 2 \mathrm{~S}$. We set out to develop technology to purify the biogas obtained at the HPTC plant of Catholic farm in Pedregal-Majes, for which a filtering system by purification with pressurized water (Scrubber) was designed, built and evaluated, to reduce the content of $\mathrm{CO} 2$ to lower values than $5 \% \mathrm{~V} / \mathrm{V}$; we work at 03 operating pressures $(6,9$ and $10 \mathrm{bar})$ combined with 03 temperatures $\left(10,15\right.$ and $\left.20^{\circ} \mathrm{C}\right)$, obtaining the best purification result, $4,3 \% \mathrm{~V} / \mathrm{V}$ of $\mathrm{CO} 2$ at $10^{\circ} \mathrm{C}$ and $10 \mathrm{bar}$ pressure. The system takes biogas, pressurizes and introduces it at the bottom of a steel tube of $0,05 \mathrm{~m}$ in diameter and $3,0 \mathrm{~m}$ in height, inside which there are $04 \mathrm{PVC}$ pipes of decreasing diameters, with perforations and spacers that increase the contact surface and therefore the absorption of $\mathrm{CO} 2$. A PSA filter system for water steam was also developed. The biogas is pressurized and introduced at the bottom through a $0,2 \mathrm{~m}$ diameter and $0,5 \mathrm{~m}$ high steel tube, inside which zeolite is found as an adsorbent. The biogas flow is controlled is controlled by valves and the filtrate is removed from the top, achieving to reduce the water steam content to $2.2 \% \mathrm{~V} / \mathrm{V}$ to 8 bar of pressure and environmental temperature.
\end{abstract}

\section{INTRODUCCIÓN}

El biogás se obtiene de la degradación de la materia orgánica (biomasa), siendo una de sus principales fuentes los residuos agrícolas; está formado por una mezcla de metano (CH4) (45 a 75\% en volumen), anhídrido carbónico (CO2) ( 25 a $45 \%$ en volumen) y otros gases como vapor de agua entre 1 a $2 \%$, nitrógeno de 1 a $5 \%$, hidrógeno entre 0 a $3 \%$, sulfuro de hidrógeno (H2S) de 0,1 a $0,5 \%$, monóxido de carbono entre 0 a $3 \%$ y oxígeno en trazas. El biogás se puede

Correspondencia:

Gonzalo Dávila-del-Carpio

gdavilad@ucsm.edu.pe utilizar como combustible por la presencia del hidrocarburo metano, pero si queremos tener un mejor rendimiento, debemos purificarlo y llevarlo a biometano, biocombustible con una concentración de $\mathrm{CH} 4$ mayor al 95\%, eliminando principalmente el contenido de $\mathrm{CO} 2$, vapor de agua y $\mathrm{H} 2 \mathrm{~S}$, éste último muy corrosivo. El biometano puede reemplazar a la gasolina y al petróleo diesel en motores de combustión interna, al propano en cocinas a gas y al gas natural en hornos y diferentes equipos industriales. El problema que se tiene es la inadecuada tecnología para la eliminación del CO2 y del vapor de agua. $[1,2,3]$.

El Perú está planteando de manera planificada, estrategias para abastecer la demanda energética derivada del crecimiento económico sostenido desde los años 90 y que se espera continúe a un ritmo similar en las siguientes décadas; dentro de ellas, las energías renovables y en especial, la bioenergía van a desempeñar un papel muy importante, siendo 
la biomasa, la fuente de energía que tiene más posibilidades de ser utilizada en sistemas aislados en los que no hay otros recursos renovables [4]. La principal fuente de recursos de biomasa en el Perú es la actividad pecuaria, seguida de la agrícola, agroindustrial, forestal y de residuos domésticos, las cuales están disponibles en cantidades superiores a 86 millones de toneladas/año [4, 5]; la biodegradabilidad y potencial metanogénico de las biomasas más representativas (estiércol de vacuno y pollo, residuos de arroz, maíz, caña de azúcar, algodón y espárragos) tienen un potencial de producción de biogás superior a 850 millones de Nm3 (metros cúbicos normalizados) por año, siendo Arequipa una de las zonas más importantes donde se produce biomasa residual [4].

Desde el 2015 el país viene impulsando el uso de biogás a través de un crecimiento sostenido en la instalación de plantas para procesar residuos orgánicos y producir biogás y biofertilizantes; en el 2016 la producción estimada de biogás fue de $26.000 \mathrm{Nm} 3$ /día, equivalente a 13.000 litros petróleo/ día, no obstante, la tecnología para el enriquecimiento de la pureza del metano y su presurización, todavía es deficiente [6]. Los sistemas de biodigestión en el país se concentran generalmente en biodigestores tipo laguna o tubulares de una sola cámara; sin embargo, desde el 2014, la Universidad Católica de Santa María (UCSM) cuenta con una planta HPTC de segunda generación, con doble cámara hidrolítica, modelo Rotaler de alta eficiencia, con una capacidad de contenedor de $30 \mathrm{~m} 3$, que produce hasta 3 veces más biogás por $\mathrm{m} 3$ de biodigestor que las convencionales, instalada en el Fundo La Católica de El Pedregal - Majes, la cual está produciendo biogás y biofertilizantes [7].

En el 2016 un equipo conformado por investigadores de la UCSM y del IIDS, ganaron un fondo de investigación del Programa Nacional de Innovación Agraria (PNIA), para desarrollar un sistema tecnológico para operar la planta HPTC y producir biometano, basándose principalmente en el uso de estiércol de vacas y cerdos, donde el combustible obtenido debía ser presurizado y utilizado en equipos industriales y agrícolas, así como para mover un tractor y un automóvil, por lo que la purificación del biogás para producir biometano, se constituía en un elemento fundamental del proyecto; previamente ya se había desarrollado tecnología para la eliminación de $\mathrm{H} 2 \mathrm{~S}$, basada en la inyección de aire al $4 \%$ en volumen, obteniéndose muy buenos resultados [8].

Al no disponer de sistemas de depuración para eliminar el CO2 y el vapor de agua del biogás producido en la planta de Majes, no se puede obtener biometano; el biogás en uso tiene un bajo poder calorífico $(18 \mathrm{MJ} / \mathrm{Nm} 3)$, haciéndolo poco competitivo con otros combustibles fósiles como el gas natural (poder calorífico $36 \mathrm{MJ} / \mathrm{Nm} 3$ ) o el gas propano (poder calorífico $48 \mathrm{MJ} / \mathrm{Nm} 3$ ). Tampoco se puede comprimir el biogás crudo o con lastre de $\mathrm{CO} 2$ y vapor de agua para almacenarlo en balones, transportarlo y simplificar, así, su uso y almacenamiento; por ello los productores tienen que almacenar el biogás a bajas presiones (20 milibares), requiriendo gasómetros de gran volumen, que encarecen y complican el diseño de las plantas de biogás. También debemos mencionar que existen otras 26 plantas de biogás en la región Arequipa, y más de 124 a nivel nacional, que requieren de una tecnología de filtrado simple y eficiente, para poder producir biometano.

Entre los métodos de purificación del biogás existen diferentes tecnologías, que aprovechan, por ejemplo, las propiedades físico-químicas de los componentes del biogás; así debemos destacar el tamaño molecular del $\mathrm{CH} 4, \mathrm{CO}$, O2, y del vapor de agua, que son $4,0,2,8,2,8$ y $3,0 \AA$ respectivamente, por lo que utilizar un adsorbente con un tamaño de poro de $3,7 \AA$ es capaz de capturar CO2, O2 y vapor de agua, pero no $\mathrm{CH} 4$, limpiando de este modo al biogás [9]. Los métodos más resaltantes son [10] :

Métodos de adsorción: basados en la captura selectiva de las impurezas del gas con materiales sólidos granulados de gran superficie específica y con la aplicación de grandes presiones; el más conocido es el sistema Pressure Swing Adsorption (PSA), donde el gas es comprimido entre $60-$ 100 psi, antes de ser introducido al sistema de adsorción, o el Temperature Swing Adsorption (TSA) [10].

Métodos de absorción: basados en transferencia de masa entre la sustancia gaseosa a depurar y un líquido absorbente que posee propiedades selectivas de absorción; se aplican principalmente para eliminar $\mathrm{H} 2 \mathrm{~S}$ y $\mathrm{CO} 2$; el solvente habitual es agua y se utilizan diversos compuestos y sistemas para la depuración, como soluciones de aminas o soluciones acuosas alcalinas; también columnas tipo duchas denominadas Scrubber, que a veces con aumento de presión pueden mejorar su eficiencia $[11,12]$.

Métodos de separación por membrana: basados en la difusión de algunos compuestos que pasan a través de una membrana selectiva; la permeabilidad del gas a través de la membrana es función de la solubilidad y la difusividad del gas en el material de la misma, permitiendo la separación del $\mathrm{CO} 2$, del H2S y del $\mathrm{H} 2$ entre otros y puede hacerse a baja o alta presión [10].

Métodos biológicos: se llevan a cabo mediante la acción de determinados microorganismos como las bacterias sulfooxidantes que convierte el $\mathrm{H} 2 \mathrm{~S}$ en azufre elemental, $\mathrm{u}$ otros que utilizan el H2S como fuente nutricional [10, 11, $12,13]$.

Últimamente también se está utilizando la criogenia [11, 12, 13].

Entre los problemas que se presentan para la purificación del biogás tenemos: dificultad para filtrar el $\mathrm{CO} 2$ debido al alto costo de los equipos y el de operación especializada (más de 12000 US\$/equipo y $0.5 \mathrm{~S} / \mathrm{Nm} 3$ de biogás filtrado, respectivamente); que los equipos disponibles en el mercado son para plantas de biogás grandes $(500 \mathrm{Nm} 3$ de biogás/día o más) y a la escasa investigación en filtrado de $\mathrm{CO} 2$ del biogás en las condiciones de Arequipa.

En este contexto, nos fijamos dos objetivos claros: el primero fue desarrollar un sistema de filtrado tipo Scrubber, que trabaje con agua presurizada, que permita filtrar el $\mathrm{CO} 2$ del biogás y reducir su contenido a valores menores al $5 \%$ $\mathrm{V} / \mathrm{V}$; el segundo objetivo estaba orientado a desarrollar un filtro del tipo de adsorción por oscilación de presión (Pressure Swing Adsorption) que trabaje con zeolita y permita filtrar el vapor de agua del biogás y reducir su contenido a valores menores al $1 \% \mathrm{~V} / \mathrm{V}$.

\section{MATERIAL Y MÉTODOS}

Para la producción del biogás por digestión anaeróbica, se utilizó la planta HPTC de segunda generación, modelo Rotaler de alta eficiencia instalada en el Fundo La Católica de El Pedregal - Majes, que tiene un contenedor de capacidad 
máxima de $30 \mathrm{~m} 3$.

Como materia prima se usó estiércol y purines de cerdo obtenidos del criadero de estos animales instalado en el Fundo; además en algunas ocasiones se añadieron residuos de viñedos.

Paralelamente se acondicionaron las instalaciones del laboratorio piloto experimental para instalar la planta de filtración y realizar las pruebas necesarias.

Luego de una intensa revisión bibliográfica, se diseñó y construyó la planta modelo a manera de un piloto experimental; en la figura $\mathrm{N}^{\circ} 1$ se observa la planta piloto diseñada para la filtración de $\mathrm{CO} 2$ y de vapor de $\mathrm{H} 2 \mathrm{O}$, que consta de los siguientes equipos y materiales:

- Un compresor de cilindro pistón y tanque de 12 bares para comprimir el biogás, con válvulas de regulación de 6 a 12 bar

- Un filtro tipo Scrubber, en tubería de metal de $0.05 \mathrm{~m}$ de diámetro y $3.0 \mathrm{~m}$ de altura, en el interior cuenta con tubos perforados de PVC con diámetros de 1", 7/8", 1/2" y $3 / 8$ ", con separadores adecuados para aumentar el área de contacto biogás - agua.

- Una Bomba de agua de $\mathrm{P}=10$ bar y caudal $15 \mathrm{~L} / \mathrm{s}$

- 02 cilindros de metal de 0,20 m. de diámetro y 0,60 m. de altura.

- Un sistema PSA, con Zeolita y válvulas de regulación entre 4 a 8 bar

- Un Sistema de enfriamiento que usa R134a, que consta de un compresor de $1 / 2 \mathrm{hp}$, el evaporador para enfriar un depósito con glycol y serpentín por donde ingresa el agua al Scrubber.

- Zeolita Micronizada - ZeoCem Eco 20,

- Analizador de gases SEWERIN, Multitec 560, que contiene sensores infrarrojos y electroquímicos para la cuantificación de $\mathrm{CH} 4, \mathrm{CO} 2, \mathrm{O} 2, \mathrm{CO}, \mathrm{H} 2 \mathrm{~S}$

- Manómetro y termómetros para aire, biogás y agua.

- Flujómetro y gasómetro, para medir caudal de agua y biogás.

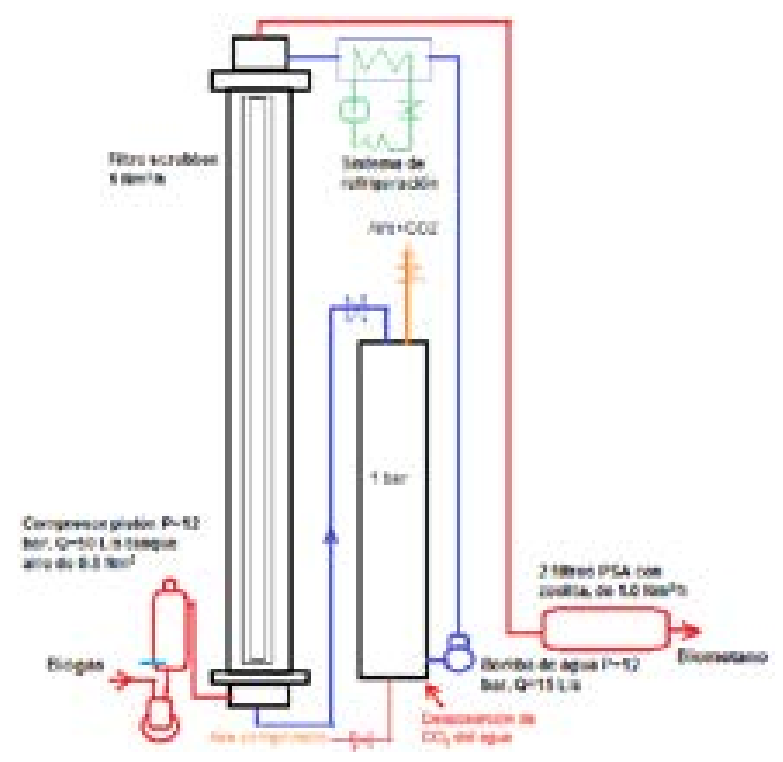

\section{Procedimiento}

a) Eliminación del CO2 a través de un sistema de filtrado por depuración tipo Scrubber:

- Una vez obtenido el biogás de la planta HPTC, se le almacenó en el contenedor de membrana y con el analizador de gases Sewerin, se cuantificó la presencia de $\mathrm{CO} 2$ y H2O, además de $\mathrm{H} 2 \mathrm{~S}$ y $\mathrm{CH} 4$; este procedimiento fue realizado en tres repeticiones.

- Durante el proceso de producción del biogás se removió el ácido sulfhídrico contenido en el biogás a través de la inyección de aire.

- Luego se diseñó y construyó el filtro tipo Scrubber, en tubería de metal de $0.05 \mathrm{~m}$ de diámetro y $3.0 \mathrm{~m}$ de altura. El filtro tiene una entrada superior por donde se abastece agua a presión, y una entrada inferior por donde se suministra el biogás. En el interior del filtro se colocaron separadores adecuados para incrementar la superficie de contacto agua:biogás. En la parte superior se colocó una salida para el biogás filtrado, y en la parte inferior una salida para el agua donde se disuelve el $\mathrm{CO} 2$.

- Luego se alimentó el biogás a través del compresor a tres presiones de operación diferentes $(6,9$ y 10 bar), combinadas con tres temperaturas distintas $(10,15 \mathrm{y}$ $\left.20^{\circ} \mathrm{C}\right)$.

- Se realizaron las mediciones del contenido de $\mathrm{CO} 2$ y $\mathrm{H} 2 \mathrm{O}$, además de $\mathrm{H} 2 \mathrm{~S}$ y $\mathrm{CH} 4$ a la entrada y a la salida del filtro tipo Scrubber. En este caso las variables dependientes fueron el contenido de $\mathrm{CO} 2(\% \mathrm{~V} / \mathrm{V})$ y metano $(\% \mathrm{~V} / \mathrm{V})$ en el biogás. Para ello se utilizó el analizador de gases Sewerin.

\section{b) Eliminación del vapor de agua a través de un sistema de filtrado PSA}

- Los dos primeros pasos fueron similares a lo ejecutado en el proceso de eliminación de $\mathrm{CO} 2$.

- Simultáneamente se diseñó, construyó y evaluó un filtro tipo PSA que se llenó con zeolita para eliminar el vapor de agua.

- El biogás que sale del filtro tipo Scrubber fue alimentado a la tubería de entrada del compresor, utilizando el presostato de la compresora y las válvulas de ingreso y salida del PSA; se reguló la presión de operación a 4, 6 y 8 bar. El caudal de alimentación fue de 1,0 Nm3 de biogás/ hora.

- Finalmente se volvió a medir la presencia de $\mathrm{CO} 2$ y $\mathrm{H} 2 \mathrm{O}$ en \% V/V con el analizador de gases Sewerin, tanto al ingreso como a la salida del filtro, así como el contenido de $\mathrm{CH} 4$.

\section{Tratamiento de datos}

- Tanto para la eliminación del CO2 utilizando el Scrubber, como para la filtración del vapor de agua a través del sistema PSA, se realizaron tres repeticiones de los ensayos y de las mediciones, concluyendo con un análisis de varianza con dos niveles de confianza, 95 y $99 \%$. 


\section{RESULTADOS}

- El diseño y construcción de todo el sistema de filtración, tanto para el Scrubber como para el PSA fue algo complicado, ya que fue armado en su integridad en la localidad de Arequipa y luego trasladado hacia el Pedregal - Majes para su montaje. Hubo que hacer muchas pruebas y adaptaciones. En la figura $\mathrm{N}^{\circ} 2$ (A) se observa el montaje de la planta del Sistema de Scrubber y PSA para la purificación del biogás.

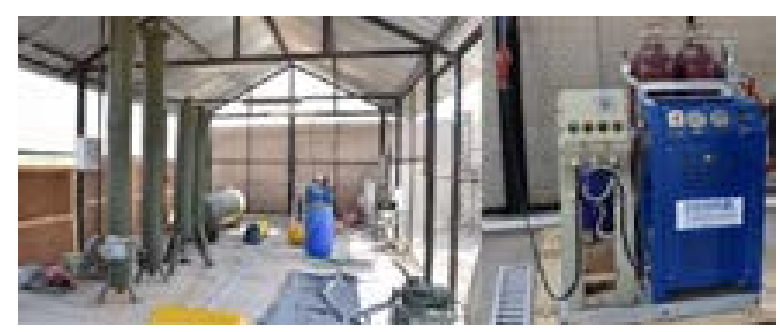

Fig. 2: A) Sistema de Scrubber y PSA para la purificación del biogás. B) Compresor para la compresión y almacenamiento en balones del biometano obtenido

- En el caso específico del Scrubber para la eliminación del $\mathrm{CO} 2$, las variables independientes manipuladas fueron dos: la temperatura, trabajándose con un ingreso del agua al scrubber de 10,15 y $20^{\circ} \mathrm{C}$; y la presión interior del Scrubber, donde trabajamos a las presiones de 6,9 y 10 bar; no se pudo trabajar a la presión proyectada de 12 bar, por la limitación de la compresora. El contenido inicial de dióxido de carbono en el biogás, se midió con el analizador de gases Sewerin y fue de $21 \% \mathrm{~V} / \mathrm{V}$ en promedio, el cual resulta un valor relativamente bajo en relación a lo reportado por la literatura. El contenido promedio de metano fue de $45,8 \% \mathrm{~V} / \mathrm{V}$. En la tabla $\mathrm{N}^{\circ} 1 \mathrm{se}$ aprecian los resultados promedio de tres determinaciones del contenido de $\mathrm{CO} 2$ en el biogás, luego del tratamiento por depuración utilizando el Scrubber a las diferentes temperaturas y presiones de ensayo.

Tabla 1: Contenido de $\mathrm{CO2}$ en el biogás luego del tratamiento por depuración en el Scrubber a diferentes temperaturas y presiones

\begin{tabular}{|c|c|c|c|c|c|c|}
\hline \multicolumn{7}{|c|}{ FILTRADO POR DEPURACIÓN (SCRUBBER) } \\
\hline $\begin{array}{c}\text { Temperatura } \\
\text { Presión }\end{array}$ & \multicolumn{2}{|c|}{$\begin{array}{l}10^{\circ} \mathrm{C} \\
6 \text { bar }\end{array}$} & \multicolumn{2}{|c|}{$\begin{array}{l}15^{\circ} \mathrm{C} \\
6 \text { bar } \\
\end{array}$} & \multicolumn{2}{|c|}{$\begin{array}{l}20^{\circ} \mathrm{C} \\
6 \text { bar } \\
\end{array}$} \\
\hline Componente & $\begin{array}{c}\% \text { volumen } \\
\text { inicial }\end{array}$ & $\begin{array}{l}\% \text { volumen } \\
\text { final }\end{array}$ & $\begin{array}{c}\% \text { volumen } \\
\text { inicial }\end{array}$ & $\begin{array}{l}\begin{array}{l}\% \text { volumen } \\
\text { final }\end{array} \\
\text { a }\end{array}$ & $\begin{array}{c}\% \text { volumen } \\
\text { inicial }\end{array}$ & $\begin{array}{l}\% \text { volumen } \\
\text { final }\end{array}$ \\
\hline $\mathrm{CO} 2$ & 21.0 & 12.3 & 21.0 & 14.0 & 21.0 & 16.2 \\
\hline Temperatura & \multicolumn{2}{|c|}{$10^{\circ} \mathrm{C}$} & \multicolumn{2}{|c|}{$15^{\circ} \mathrm{C}$} & \multicolumn{2}{|c|}{$20^{\circ} \mathrm{C}$} \\
\hline Presión & \multicolumn{2}{|c|}{9 bar } & \multicolumn{2}{|c|}{9 bar } & \multicolumn{2}{|c|}{9 bar } \\
\hline Componente & $\begin{array}{c}\% \text { volumen } \\
\text { inicial }\end{array}$ & $\begin{array}{l}\% \text { volumen } \\
\text { final }\end{array}$ & $\begin{array}{c}\% \text { volumen } \\
\text { inicial }\end{array}$ & $\begin{array}{l}\% \text { volumen } \\
\text { final }\end{array}$ & $\begin{array}{c}\% \text { volumen } \\
\text { inicial }\end{array}$ & $\begin{array}{l}\text { \% volumen } \\
\text { final }\end{array}$ \\
\hline $\mathrm{CO} 2$ & 21.0 & 6.9 & 21.0 & 9.2 & 21.0 & 11.6 \\
\hline Temperatura & \multicolumn{2}{|c|}{$10^{\circ} \mathrm{C}$} & \multicolumn{2}{|c|}{$15^{\circ} \mathrm{C}$} & \multicolumn{2}{|c|}{$20^{\circ} \mathrm{C}$} \\
\hline Presión & \multicolumn{2}{|c|}{10 bar } & \multicolumn{2}{|c|}{$10 \mathrm{bar}$} & \multicolumn{2}{|c|}{10 bar } \\
\hline Componente & $\begin{array}{l}\% \text { volumen } \\
\text { inicial }\end{array}$ & $\begin{array}{l}\% \text { volumen } \\
\text { final }\end{array}$ & $\begin{array}{l}\% \text { volumen } \\
\text { inicial }\end{array}$ & $\begin{array}{l}\% \text { volumen } \\
\text { final }\end{array}$ & $\begin{array}{l}\% \text { volumen } \\
\text { inicial }\end{array}$ & $\begin{array}{l}\% \text { volumen } \\
\quad \text { final }\end{array}$ \\
\hline $\mathrm{CO} 2$ & 21.0 & 4.3 & 21.0 & 6.3 & 21.0 & 8.5 . \\
\hline
\end{tabular}

A las tres presiones ensayadas podemos apreciar que a presión constante, la eficacia de la filtración de $\mathrm{CO} 2$ en el Scrubber disminuye al aumentar la temperatura; sin embargo, para las tres temperaturas utilizadas, a medida que aumentamos la presión, el CO2 es eliminado con mayor eficacia.

Los datos fueron procesados estadísticamente a través del análisis de varianza, encontrándose diferencias estadísticas significativas tanto para el nivel de confianza de $95 \%$ y el de $99 \%$, pudiendo apreciarse que a la temperatura de $10^{\circ} \mathrm{C}$ y a una presión de 10 bar se obtuvieron los mejores resultados.

- En el caso del tratamiento del biogás para la eliminación del vapor de agua, se diseñó, construyó y evaluó un sistema de filtrado de Adsorción por Oscilación de Presión (Pressure Swing Adsorption, PSA). Se evaluaron 3 presiones de operación (4, 6 y 8 bar) con un caudal de alimentación de $1 \mathrm{Nm} 3$ / hora.

El sistema toma biogás, lo presuriza e introduce por la parte inferior de un tubo de acero de $0,20 \mathrm{~m}$ de diámetro y 0,5 $\mathrm{m}$ de altura, en cuyo interior se encuentra la zeolita. El flujo de biogás es controlado a través de válvulas y una vez filtrado se retira por la parte superior.

El contenido inicial de agua en el biogás fue medido con el analizador de gases Sewerin, obteniéndose un valor promedio de $18,4 \%$, valor elevado en relación a lo reportado por la literatura, lo que le da características particulares al biogás obtenido en la planta de Majes.

Los resultados se muestran en la tabla $\mathrm{N}^{\circ} 2$ y se aprecia que la reducción máxima de contenido de vapor de agua se alcanza con la presión de 8 bar y a temperatura ambiente, lográndose un contenido de $2,2 \%$ de vapor de agua en volumen.

Tabla 2: Contenido de agua en el biogás luego del tratamiento con PSA a diferentes presiones

\begin{tabular}{ccc}
\hline \multicolumn{3}{c}{ Pressure Swing Adsorption (PSA), con zeolite } \\
\hline $\begin{array}{c}\text { Presión Interna en el PSA } \\
\text { COMPONENTE }\end{array}$ & \% bar \\
Agua & 18.4 & 8.1 \\
\hline \multicolumn{2}{c}{6 de volumen inicial } & $\%$ de volumen final \\
\hline $\begin{array}{c}\text { Presión Interna en el PSA } \\
\text { COMPONENTE }\end{array}$ & \% de volumen inicial $\%$ de volumen final \\
Agua & 18.4 & 6.3 \\
\hline Presión Interna en el PSA & 8 bar \\
COMPONENTE & $\%$ de volumen inicial $\%$ de volumen final \\
Agua & 18.4 & 2.2 \\
\hline
\end{tabular}

Nota: Los datos reportados son promedio de tres determinaciones

Los datos fueron procesados estadísticamente, a través del análisis de varianza, determinando un valor de $\mathrm{F}=$ 748,09 , muy por encima de los valores de la $\mathrm{F}$ teórica para 2 y 6 grados de libertad, a un nivel de confianza del $95 \%$ de 5,14; a un nivel de confianza del $99 \%$ el valor de F fue de 10,9 , por consiguiente se rechaza la hipótesis nula y se concluye que existen diferencias significativas cuando el biogás es sometido a un proceso de filtrado por PSA a tres presiones diferentes, observándose una mayor eliminación 
de vapor de agua a 8 bar de presión

Además existe una buena correlación lineal con un valor de $\mathrm{r}=0,9756$ entre la presión de operación en el filtro y el contenido final de vapor de agua en el biogás.

- Debemos mencionar que luego de la purificación del biogás, el biometano obtenido llegó a un contenido promedio de $85 \%$ en volumen de metano, que de acuerdo a lo recomendado por la literatura no es lo ideal.

\section{DISCUSIÓN}

El filtrado de $\mathrm{CO} 2$ utilizando scrubbers se basa en la absorción física del $\mathrm{CO} 2$ al utilizar agua como solvente. La solubilidad del $\mathrm{CO} 2$ en agua es varias veces mayor que la solubilidad del $\mathrm{CH} 4$; la temperatura de operación utilizada en la investigación varió entre 10 a $20^{\circ} \mathrm{C}$; a $20^{\circ} \mathrm{C}$, la solubilidad del $\mathrm{CO} 2$ es de $1.65 \mathrm{~g}$ por $\mathrm{kg}$ de agua, y del $\mathrm{CH} 4$ es de $0.023 \mathrm{~g}$ por kg de agua [14], lo cual asegura una mayor disolución y por lo tanto transferencia del CO2 hacia el agua que el metano. Las dos variables más importantes en el scrubber son la presión y la temperatura del agua, en menor escala la longitud del scrubber [13].

Chen et al., en el 2014 demostraron que la eliminación de $\mathrm{CO} 2$ se incrementa de $34,6 \%$ a 94,2 \% cuando la relación líquido/gas aumenta de 0,14 a 0.50 . Otros estudios como el realizado por Beil y Hoffstede en el 2010, trabajaron en un rango de operación de presión entre 6 y 12 bar, demostrando que a cualquier temperatura, la absorción de $\mathrm{CO} 2$ aumenta al incrementarse la presión, tal como sucede en esta investigación.

Rasi et al., en el 2007 utilizaron presiones entre 20 y 30 bar con buenos resultados, logrando una reducción a niveles de $3.2 \%$ en volumen de $\mathrm{CO} 2$, y el $\mathrm{H} 2 \mathrm{~S}$ disminuyó hasta valores al límite de detección; para ello fue necesario un sistema de refrigeración.

Podemos apreciar que los resultados que obtuvimos son corroborados por las diferentes investigaciones reportadas, que a mayor presión y menor temperatura la absorción del CO2 es mayor; los límites de estos parámetros dependen de la simplicidad de la planta, menores costos de equipos y mantenimiento que repercuten en los costos del biometano producido. Sin embargo, a mayor escala de producción de biometano, es necesario corregir algunos aspectos de la planta, como longitud del scrubber, usar la desabsorción para recircular parte del agua, usar un compresor de dos etapas con inter-enfriamiento para el biogás crudo y aumentar ligeramente la presión entre 15 y 20 bar.

En el presente estudio, se aprecia que a la temperatura de $10^{\circ} \mathrm{C}$ y a una presión de $10 \mathrm{bar}$, los niveles de $\mathrm{CO} 2$ alcanzan un valor promedio de $4,3 \%$ en volumen, por debajo del $5 \%$ que se fijó como objetivo, lo cual resulta bueno para la calidad del biometano obtenido.

En cuanto a la eliminación del vapor de agua, este puede removerse enfriando el biogás [17]. La cantidad de vapor de agua en el biogás depende de la presión y temperatura, variables utilizadas en este trabajo. El punto de rocío del biogás es de $1.7 \mathrm{oC}$, y contiene típicamente $10 \%$ en volumen de vapor de agua a 43 oC, $5 \%$ a 32 oC y $1 \%$ a 4 oC [18]. Es decir, a medida que disminuye la temperatura, disminuye el contenido de vapor de agua.

Otras investigaciones refieren que a 32 oC el biogás contiene $32 \mathrm{~g}$ agua/ $\mathrm{Nm} 3$; la refrigeración del biogás puede efectuarse utilizando la temperatura de recursos adyacentes (suelo, agua) o por sistemas de refrigeración. El vapor de agua también puede removerse con Scrubbers conteniendo glicol o por adsorción en silicatos, carbón activado o cribas moleculares como la zeolita [19].

La Pressure Swing Adsorption (PSA) es una tecnología utilizada para separar el vapor de agua del biogás, basada en las características moleculares y la afinidad del vapor de agua por la zeolita $\mathrm{u}$ otros adsorbentes [12]. Opera a temperatura ambiente y a presiones entre 4 a 7 bar. Luego de adsorber el vapor de agua, el proceso cambia al reducir la presión a presión ambiente y desadsorbe el vapor de agua [20]. El gas se presuriza y es conducido a través de una columna llena con zeolita (CAS \# 63231-67-4). Por lo general se utilizan dos columnas en paralelo: una columna adsorbe el agua, mientras que la otra regenera la zeolita; la regeneración se logra por evaporación del agua a través de la descompresión (a presión atmosférica) y calefacción $\left(100\right.$ a $\left.200{ }^{\circ} \mathrm{C}\right)$ [21].

Cuando se utiliza un sistema PSA, se sabe que la variable principal es la presión, sin embargo, tiene que ver también el tamaño de poro del adsorbente, el caudal y/o velocidad del gas dentro del PSA y la temperatura. Estas variables están en función al diseño tanto del PSA como de toda la planta incluyendo el scrubber, el compresor, las caídas de presión que son función de la longitud y diámetro de las tuberías y los costos de cada uno de los elementos de la planta. Se observa que a mayor presión del gas, la adsorción por parte de la zeolita es más eficiente, sin embargo, el límite de presión tiene que ver con costos del compresor y operación del mismo, el que se incrementa si la presión sube.

El biometano que se obtuvo en la planta, cumple con las especificaciones planteadas para el $\% \mathrm{CO} 2<$ a $5 \%$ $\mathrm{V} / \mathrm{V}$; en cuanto al vapor de agua la meta fue $\% \mathrm{H} 2 \mathrm{O}<\mathrm{a}$ $1 \% \mathrm{~V} / \mathrm{V}$, sin embargo, se observa que a 8 bar se obtuvo un $2.2 \% \mathrm{~V} / \mathrm{V}$. Este no es un problema, ya que a la presión de 8 bar, el biometano obtenido debe ingresar a un tanque, y a condiciones ambientales el agua condensa y debe retirarse a través de una válvula por la parte inferior. En realidad, si el scrubber trabaja a 10 bar, el PSA trabajará a 9.5 bar, por lo tanto el \% de vapor de $\mathrm{H} 2 \mathrm{O}$ será menor, la presión del tanque de biometano estará por los 9 bar, es decir con mayor energía para los siguientes procesos, como son la compresión a presiones más altas y su transporte y uso a 17 bares y 250 bares, respectivamente, tal como está programado para sus aplicaciones futuras, donde nuevamente habrá condensación de las pequeñas trazas de vapor de agua, logrando de esa manera valores menores a $1 \% \mathrm{~V} / \mathrm{V}$ en el biometano.

Los costos del biometano a los que se llega con esta planta, son costos propios de implementación y costos operativos (mano de obra y mantenimiento); estos no fueron plenamente analizados, sin embargo, la depreciación de la planta se realiza en 10 años, y considerando los porcentajes de operación y mantenimiento, se puede demostrar que el costo del biometano incluyendo el costo del biogás es menor a $0.18 \mathrm{~S} / \mathrm{Nm} 3$ de biogás procesado.

Para plantas mayores, otra forma de reducir costos es logrando la automatización de cada uno de los procesos, en el compresor, scrubber, PSA, bombas. Si bien aumenta el costo de la planta, se reducen grandemente los costos de operación y mantenimiento.

En conclusión, se diseñó, construyó y validó una planta a escala reducida, con un sistema de filtrado por depuración, 
tipo Scrubber, para absorber el CO2 del biogás y un sistema PSA con zeolita como adsorbente de vapor de agua, lo que nos permitió reducir la presencia de estos contaminantes en el biometano a proporciones aceptables.

\section{AGRADECIMIENTOS}

- Queremos agradecer el financiamiento otorgado inicialmente por el Centro Interdisciplinario de Investigación e Innovación de la Universidad Católica de Santa María y a partir del año 2015, por el Vicerrectorado de Investigación de la Universidad.

- Igualmente nuestro reconocimiento a los funcionarios y personal del Fundo La Católica de Majes en El Pedregal, quienes nos brindaron el acceso a la Planta de Biogás instalada en dicha localidad.

\section{REFERENCIAS BIBLIOGRÁFICAS}

1. Kanokwan Boe. (April 2010). Seminar on Digestion of Sludge. Finnish Environment Institute. Monitoring, control and optimization of the Biogás process. Bioenergy Research Group. Department of Environmental Engineering. Technical University of Denmark

2. Peña, G. \& Salazar, I. (2009). Digestión Anaeróbica aplicada al aprovechamiento de Residuos Agroindustriales. Arequipa: FINCyT.

3. Ponce Mallea, Marco. (2015). Energías Renovables: Biogás (Review). Primera Edición, Universidad Católica de Santa María. Arequipa.

4. Hidalgo, Dolores y Equipo del Proyecto BIOMETRANS (2018). Producción de biometano para combustible de transporte a partir de residuos de biomasa: Diagnóstico de los recursos de biomasa disponibles en Iberoamérica. Programa Iberoamericano CYTED - España. ISBN/ ISSN: 978-84-09-07532-4.

5. Assureira-Espinoza, E. G. y Assureira-Espinoza M. A., (2015), Potencial energético de la biomasa residual en el Perú, Lima, Perú: Pontificia Universidad Católica del Perú.

6. Reategui, J., Peña, G., Cárdenas, L., Castro, J., Mejía, F., Mestas, S. \& Roque, F. (2018). Manual de Producción y Uso de Biometano Presurizado a Baja y Alta Presión. Arequipa, Perú: UCSM.

7. Peña, G. \& Soldevilla, S. (2012). Desarrollo de tecnología para el procesamiento eficiente del biogás en la región Arequipa. Arequipa.

8. Hidalgo Dolores y Equipo del Proyecto BIOMETRANS (Junio 2018). Producción de biometano para combustible de transporte a partir de residuos de biomasa: Actualización del estado del conocimiento en aprovechamiento de biomasa para producción de biogás en la Región Iberoamericana. . Programa Iberoamericano CYTED - España. ISBN/ISSN: 97884-09-07533-1.

9. Sun, Q., Li, H., Yan, J., Liu, L., Yu, Z., \& Yu, X. (2015). Selection of appropriate biogas upgrading technology-a review of biogas cleaning, upgrading and utilisation. Renewable and Sustainable Energy Reviews, 51, 521532. http://doi.org/10.1016/j.rser.2015.06.029.

10. Llanez H., Morís M. A., González Aspíroz L. Y
González E. (Marzo 2010). Estudios de viabilidad de sistemas de purificación y aprovechamiento de biogás. Capítulo 1: Caracterización, purificación y control de biogás. PSE PROBIOGÁS. Desarrollo de sistemas sostenibles de producción y uso de biogás industrial en España.

11. Pao Chi Chen, C.F. Huang, Hsiao-Wei Chen, MingWei Yang, Chih-Ming Tsao. (2014). Capture of $\mathrm{CO} 2$ from Coal-fired Power Plant with $\mathrm{NaOH}$ Solution in a Continuous Pilot-scale Bubble-column Scrubber. Energy Procedia, Volume 61, pages 16601664. ISSN 1876-6102, https://doi.org/10.1016/j. egypro.2014.12.186.

12. Niesner, J., Jecha, D., \& Stehlík, P. (2013). Biogas upgrading technologies: State of art review in european region. Chemical Engineering Transactions, 35, 517522. http://doi.org/10.3303/CET1335086

13. Chen, P. C., Huang, C. F., Chen, H. W., Yang, M. W., \& Tsao, C. M. (2014). Capture of CO2 from coal-fired power plant with $\mathrm{NaOH}$ solution in a continuous pilot-scale bubble-column scrubber. Energy Procedia, 61(1), 1660-1664. http://doi.org/10.1016/j. egypro.2014.12.186.

14. Couvert, A., Sanchez, C., Laplanche, A., Renner, C., \& Levasseur, J. P. (2008). Design of a new compact scrubber for deodorisation. Chemical Engineering and Processing: Process Intensification, 47(9-10), 17931798. http://doi.org/10.1016/j.cep.2007.10.006.

15. Beil, M. and Hoffstede, U. (2010). Guideline for the implementation and operation of biogás upgrading systems. Kassel: Fraunhofer IWES.

16. Rasi, S., Lehtinen, J. and Rintala, J. (2010). Determination of organic silicon compounds in biogás from wastewater treatment plants, landfills and codigestion plants. Renewable Energy. Vol. 35, pp. 2666 -2673 .

17. Mohamad, I.N., Rohani, R. Vervaeren, H. (2017). An overview of Gas-Upgrading Technologies for Biohydrogen produced for treatment of Palm Oil mill efluent. Journal of Engineering Science and Technology. Vol. 12, $\mathrm{N}^{\circ}$ 3: 725-755. School of Engineering, Taylor University, 12(2): 1-33.

18. Esteves, I.A.A.C., Souza, G., Silva, R.J.S., Ribeiro, R.P.P., Eusébio, M.F., Mota J.P.B. (2016). A sentitive method approach for Chromatographic Analysis of Gas streams in separation processes base don columns packed with an Adsorbent Material. Advances in Materials Science and Engineering. Article ID 3216267. http://dx.doi.org/10.1155/2016/3216267,2016.

19. TUV (2012). Biogas to Biomethane Technology review. Vienna University of Technology. May 1-15. Retrieved from http://www.aile.asso.fr/wp-content/ uploads/2012/06/wp3-1-1_technologyreview_english. pdf

20. Yousef, A.M.I., Eldrainy, Y.A., El-Maghlany, W.M., Attia, A. (2016). Upgrading Biogas by a lowtemperature $\mathrm{CO} 2$ removal technique. Alexandria Engineering Journal. 55 (2): 1143-1150. http:/doi. org/10.2016/j.aej.2016.03.026.

21. Ryckebosch, E., Drouillon, M. and Vervaeren Han. (2011). Techniques for transformation of biogas to biomethane. Biomass and Bioenergy 35(5):1633-1645. DOI: 10.1016/j.biombioe.2011.02.033. 